Inflammatory bowel disease

\section{A place for TACE}

\section{S J H van Deventer}

\section{Inhibition of tumour necrosis factor $\alpha$ converting enzyme as a new therapeutic target in inflammatory bowel disease}

V ertebrates, and humans are no exception, have developed potent innate and adaptive immune systems that deal with potential lethal encounters with microorganisms, which are critically dependent on the biological effects of proinflammatory cytokines such as interleukin 12, interleukin 18 interferon $\gamma$, and tumour necrosis factor $\alpha$ (TNF- $\alpha)$. The pivotal role of these cytokines is underscored by the observation that neutralisation of TNF- $\alpha$ in patients with Crohn's disease may convert latent tuberculosis into a potentially lethal disseminated form. ${ }^{1}$ Thus proinflammatory cytokines are necessary for survival of the human species, but as they may cause significant inflammatory damage, their production and secretion need to be tightly regulated.

Indeed, the production and secretion of TNF- $\alpha$ are controlled at multiple checkpoints, no doubt in order to prevent unrestrained inflammation and tissue damage. TNF- $\alpha$ is translated as a precursor protein which contains an unusually long signal peptide that anchors the protein to the outer membrane. During local and systemic inflammatory reactions, membrane bound TNF- $\alpha$ is cleaved extracellularly by a specific zinc dependent metalloprotease that has been designated TNF- $\alpha$ converting enzyme (TACE), yielding the soluble homotrimeric form of TNF- $\alpha$ that can act either as a compartmentalised or circulating cytokine. It was initially thought that cleavage of TNF- $\alpha$ constituted a major final road block for TNF- $\alpha$ production but subsequently it was recognised that membrane expressed TNF- $\alpha$ is biologically active as a homotrimer during cellcell contact by interacting with both p55 and p75 TNF receptors. ${ }^{2}$

TACE is member of the ADAM (a disintegrin and metalloproteinase) family of cell surface proteases, which are induced during inflammation, ${ }^{3}{ }^{4}$ and has attracted a lot of attention because small molecules that can be relatively easily manufactured are known to effectively block the function of metalloproteases. In this issue of Gut, Brynskov and colleagues $^{5}$ report that in the normal colon mucosa, mononuclear as well as epithelial cells express bioactive TACE, and that TACE activity is increased in mucosal biopsies from patients with active ulcerative colitis but not in Crohn's disease [see page 37]. TACE activity was blocked ex vivo by metalloprotease/ TACE inhibitors or zinc chelating agents, but not by trocade, a broad spectrum metalloprotease that is known not to affect TACE activity.

Is TACE an attractive therapeutic target in inflammatory bowel disease? Clearly, inhibition of TACE will lead to a reduction in the amount of secreted TNF- $\alpha$ but the number of membrane bound TNF- $\alpha$ molecules is not significantly altered. ${ }^{6}$ Soluble and membrane bound forms have different biological functions and this has been clearly demonstrated by studying the phenotype of mice that were engineered to express a form of $\mathrm{T}$ lymphocyte targeted TNF- $\alpha$ that cannot be cleaved by TACE or other metalloproteases. $^{7}$ As a consequence, these mice exclusively express membrane bound TNF- $\alpha$. Such mice are still susceptible to the development of a wide range of $\mathrm{T}$ lymphocyte mediated inflammatory diseases, including arthritis, hepatitis, and encephalitis, clearly indicating an important role of membrane bound TNF- $\alpha$ in the pathogenesis of this diseases.

It should also be noted that none of the currently available TACE inhibitors is entirely specific, and they also affect the function of other metalloproteases as well as cleavage of multiple human membrane expressed molecules, including both TNF receptors. ${ }^{68}$ Normally, TNF receptors are cleaved during inflammation, and this has a TNF- $\alpha$ regulatory function because further TNF- $\alpha$ signalling is inhibited and because soluble TNF receptors retain the ability to bind and neutralise soluble TNF- $\alpha$. In humans, TACE inhibitors indeed interfere not only with TNF- $\alpha$ production but also with the shedding of TNF- $\alpha$ receptors. ${ }^{6}$ This mechanism has been postulated to explain the paradoxical finding that treatment of experimental arthritis with a TACE inhibitor increased rather than inhibited the inflammatory response." Another study reported that a TACE inhibitor reduced the severity of TNBS induced colitis in mice but this was not associated with a reduction in TNF- $\alpha$ production, strongly suggesting that inhibition of metalloproteases other than
TACE was the mechanism of action. ${ }^{10}$ Because several non-TACE metalloproteases have been strongly implicated in tissue damage and remodelling during inflammatory bowel disease, ${ }^{11}$ this would still be an interesting therapeutic intervention but it should be noted that long term non-specific inhibition of metalloproteases could result in (ectopic) collagen deposition and fibrosis, and this may lead to stenosis.

In conclusion, TACE inhibitors block the release of TNF- $\alpha$ by mononuclear cells but not the membrane expressed form. Therefore, these compounds act in a very different manner than TNF- $\alpha$ binding molecules that also target membrane bound TNF- $\alpha$, resulting in apoptosis of TNF- $\alpha$ producing cells. ${ }^{12}$ Inhibition of TNF- $\alpha$ release by TACE inhibitors is expected to have anti-inflammatory effects, but in experimental models of $\mathrm{T}$ lymphocyte mediated diseases, expression of membrane bound TNF- $\alpha$ is sufficient for induction of disease. Finally, none of the currently available TACE inhibitors is entirely specific and inhibition of non-TACE metalloproteases may cause unexpected side effects.

Gut 2002;51:5-6

\section{Author's affiliation}

S J H van Deventer, Department of Gastroenterology, Academic Medical Centre, Amsterdam, the Netherlands:

s.j.vandeventer@amc.uva.nl

\section{REFERENCES}

1 Keane J, Gershon S, Wise RP, et al. Tuberculosis associated with infliximab, a tumor necrosis factor alpha-neutralizing agent N Engl J Med 2001;345: 1098-104.

2 Alexopoulou L, Pasparakis M, Kollias G. A murine transmembrane tumor necrosis factor (TNF) transgene induces arthritis by cooperative p55/p75 TNF receptor signaling. Eur J Immunol 1997;27:2588-92.

3 Moss ML, Jin SL, Milla ME, et al. Cloning of a disintegrin metalloproteinase that processes precursor tumour-necrosis factor-alpha (published erratum appears in Nature 1997;386:738). Nature 1997;385:733-6.

4 Black RA, Rauch CT, Kozlosky CJ, et al. A metalloproteinase disintegrin that releases tumour-necrosis factor-alpha from cells. Nature 1997;385:729-33.

5 Brynskov J, Foegh P, Pedersen G, et al. Tumour necrosis factor $\alpha$ converting enzyme (TACE) activity in the colonic mucosa of patients with inflammatory bowel disease. Gut 2002;51:37-42

6 Dekkers PE, Lauw FN, ten Hove $T$, et al. The effect of a metalloproteinase inhibitor (GI5402) on tumor necrosis factor-alpha (TNF-alpha) and TNF-alpha receptors during human endotoxemia. Blood 1999;94:2252-8.

7 Georgopoulos S, Plows D, Kollias G. Transmembrane TNF is sufficient to induce localized tissue toxicity and chronic inflammatory arthritis in transgenic mice. $J$ Inflamm 1996;46:86-97.

8 Dekkers PE, ten Hove T, Lauw FN, et al. The metalloproteinase inhibitor $\mathrm{Gl} 5402$ inhibits endotoxin-induced soluble CD27 and CD16 release in healthy humans. Infect Immun 2000;68:3036-9.

9 Williams LM, Gibbons DL, Gearing A, et al. Paradoxical effects of a synthetic 
metalloproteinase inhibitor that blocks both p55 and p75 TNF receptor shedding and TNF alpha processing in RA synovial membrane cell cultures. J Clin Invest 1996:97:2833-41.

10 Sykes AP, Bhogal R, Brampton C, et al. The effect of an inhibitor of matrix metalloproteinases on colonic inflammation in a trinitrobenzenesulphonic acid rat model of inflammatory bowel disease. Aliment Pharmacol Ther 1999:13:1535-42.

11 Heuschkel RB, MacDonald TT, Monteleone $G$, et al. Imbalance of stromelysin-1 and TIMP-1 in the mucosal lesions of children with inflammatory bowel disease. Gut 2000:47:57-62

12 Lugering A, Schmidt M, Lugering $N$, et al. Infliximab induces apoptosis in monocytes from patients with chronic active crohn's disease by using a caspase-dependent pathway. Gastroenterology 2001;121:1145-57.

\section{A long hard look at Dukes' B G T Williams}

\section{Identification of prognostic pathological factors in Dukes' B colon cancer}

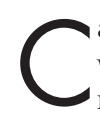
arcinoma of the colon is somewhat of a Cinderella among colorectal cancers, and during the last few years its ugly sister, carcinoma of the rectum, has hogged the limelight. This has been largely due to the (deserved) attention that has been paid to reducing local recurrence in rectal cancer through better preoperative imaging, surgical technique, and adjuvant chemoradiotherapy. However, cancer of the colon remains a highly lethal tumour. There is little controversy that it is best treated initially by surgical resection, that patients with completely resected Dukes' A tumours of usual histological type require no more treatment, and that patients found to have Dukes' C tumours after histological examination of the resection specimen should be offered adjuvant chemotherapy. ${ }^{1}$ For the $40 \%$ or so of colonic cancers that are Dukes' B, however, there is great uncertainty surrounding the place of such chemotherapy, as has been highlighted in the recently published revised Guidelines for the management of colorectal cancer (2001) from the Association of Coloproctology of Great Britain and Ireland (ACP). ${ }^{2}$ Part of the reason for this is that the Dukes' $\mathrm{B}$ category encompasses a wide range of tumours, from those that have just penetrated the muscular coat of the bowel wall to widely infiltrative neoplasms that show extensive extramural local spread, and it would not be surprising that the benefit of postoperative chemotherapy might vary in parallel with this. Oncologists have often spoken informally about "good Bs" and "bad Bs" and the new ACP guidelines propose certain pathological criteria that could be used to identify a subgroup of cases that might benefit from chemotherapy.
Another issue contributing to the uncertainty is the quality of the pathological examination of resection specimens on which the staging of colon cancers and the identification of adverse prognostic features is based. If this is not uniform within and between clinical trials then outcome comparisons may be meaningless. For example, inadequate sampling of the primary tumour will understage some Dukes' B cancers as Dukes' A tumours while inadequate lymph node sampling will understage some Dukes' C tumours as Dukes' Bs. Furthermore, pathological examination of cancers in most multicentre clinical trials is undertaken by a large number of different pathologists whose interpretation of pathological features is subject to interobserver variation. It is only recently that there has been any attempt at ensuring uniformity of pathological approach and review of slides within trials, and even this does not completely guarantee the quality of specimen dissection. The situation is further confused by pooling of patients and grouping of trials in different analyses (IMPACT B2 1999, Mamounas et al 1999). ${ }^{3}$

Given this background, the paper by Petersen and colleagues ${ }^{5}$ from Gloucester in this issue of Gut is of immense importance to pathologists, surgeons, and oncologists, and to the designers of future clinical trials of colon cancer [ see page 65]. It describes a prospective study of 268 unselected Dukes' B colon cancers (from a total population of 673 colon cancers and 377 rectal cancers) treated by primary resection in a single centre that have been meticulously dissected and characterised by a single gastrointestinal histopathologist. The quality is assured by the fact that the mean number of tumour blocks examined was 5.7 and the mean lymph node harvest was 21.3. We can therefore be confident that these are genuine Dukes' $\mathrm{B}$ cases. Equally rigorous methods were used for assessing the various pathological features and for assessing their prognostic impact by univariate and multivariate analysis. The results clearly show that four factors, namely tumour perforation, peritoneal involvement, venous spread, and surgical margin involvement, when carefully assessed in this way, are independently highly predictive of outcome. Dukes' B tumours with none of these adverse features have a similar prognosis to Dukes' A tumours while the presence of tumour perforation or any two of the other three factors reduces the five year survival to $50 \%$. Interestingly, these are four of the five factors that were proposed in the ACP guidelines (without any evidence being presented) as possible indicators for considering adjuvant chemotherapy in colon cancer (the fifth was poorly differentiated histology) and Petersen et al's paper adds much weight to these proposals. It would have been interesting to know whether the individual pathological features further predicted the pattern of disease relapse (for example, did tumour perforation and serosal involvement predict peritoneal carcinomatosis, did venous spread predict distant metastasis, and did margin positivity predict local recurrence?), because future refinement of adjuvant postoperative therapies might allow prevention of each of these complications to be more specifically targeted.

None of the four prognostic pathological factors comes as any great surprise to pathologists but there are three features of detail that are of particular note. Firstly, Petersen et al found that not only did extramural venous invasion have prognostic significance but submucosal venous invasion also, which is at variance with the findings of Talbot et al's classic paper in rectal cancer. ${ }^{6}$ It is difficult to conceive of an anatomical reason why things should be different between the colon and rectum, but if this finding is confirmed, pathologists will have to start paying attention to recording submucosal as well as extramural venous involvement when reporting colon cancer, and the Royal College of Pathologists' Minimum Dataset for cancer reports modified accordingly. Secondly, the Gloucester group found that not only is involvement of the nonperitonealised surgical margin (the socalled circumferential resection margin) 
an important prognostic factor but that acute suppurative inflammation at this margin that is in continuity with the tumour itself has similar importance. Such a situation is most likely to occur in sigmoid colon cancers that are accompanied by diverticulitis. Amazingly, no previous analysis of prognostic features in colon cancer has examined the significance of circumferential margin involvement, and although it appears to be infrequent in colon cancer, the evidence presented indicates that pathologists who have not assessed it previously in colon cancer should start doing so. The methods used are exactly the same as those that are now routinely used in rectal cancer, where the "bare" retroperitoneal or mesocolic surgical surface (which occupies about half the circumference of the bowel in the caecum, the proximal ascending colon, and the distal sigmoid colon but is barely existent in the transverse colon) is painted with a marker and the specimen serially sliced transversely to identify where the tumour or any associated suppuration is closest. Thirdly, Petersen et al's paper highlights the importance of meticulously searching for peritoneal involvement in colonic cancer by taking at least two blocks of the tumour where it is closest to the peritoneal surface. The findings reinforce previous studies from the Gloucester group that have highlighted the immense prognostic power of this feature ${ }^{8}$ and give further impetus to the consideration of intraperitoneal therapy for patients with such tumours.

Petersen et al's paper probably represents the best available prognostic analysis of pathological variables in Dukes' B colon cancer. It represents a labourious study of the highest quality achievable, carefully executed by a highly motivated expert pathologist. Its findings deserve wide recognition and integration into clinical practice.

Gut 2002;51:6-7

\section{Author's affiliation}

G T Williams, Department of Pathology, University of Wales College of Medicine, Cardiff, CF14 4XN; williamsgt@cf.ac.uk

\section{REFERENCES}

1 Dube $S$, Heyen F, Jenicek M. Adjuvant chemotherapy in colorectal carcinoma: results of a meta-analysis. Dis Colon Rectum 1997:40:35-41.

2 Association of Coloproctology of Great Britain and Ireland. Guidelines for the management of colorectal cancer (2001). London: Association of Coloproctology of Great Britain and Ireland, 2001.

3 IMPACT B2 investigators. Efficacy of adjuvant fluorouracil and folinic acid in B2 colon cancer. J Clin Oncol 1999; 17:1356-63.

4 Mamounas $\mathrm{E}$, Wieand S, Wolmark N, et al. Comparative efficacy of adjuvant chemotherapy in patients with Dukes' B versus Dukes' $C$ colon cancer: results from four national surgical adjuvant breast and bowel project adjuvant studies. J Clin Oncol project adjuvant studies

5 Petersen VC, Baxter KJ, Love SB, et al. Identification of objective pathological prognostic determinants and models of prognosis in Dukes' B colon cancer. Gut 2002;51:65-9

6 Talbot IC, Ritchie S, Leighton MH, et al. The clinical significance of invasion of veins by rectal cancer. Br J Surg 1980;67:439-42.

7 Burroughs, Williams GT. ACP Best Practice No 159: Examination of large intestine resection specimens. J Clin Pathol 2000:53:344-9.

8 Shepherd NA, Baxter KJ, Love SB. The prognostic importance of peritoneal involvement in colonic cancer: a prospective evaluation. Gastroenterology 1997;112:1096-102.

\section{Weighty issues in hepatitis $C$}

\section{J Heathcote}

\section{Simple lifestyle changes, inducing weight reduction over three months, can potentially reduce both the morbidity and mortality related to hepatitis $C$ infection}

$\mathrm{T}$ he paper by Hickman and colleagues ${ }^{1}$ in this issue of Gut [see page 89] is important because it shows that simple lifestyle changes inducing weight reduction over three months can lead to a significant reduction in hepatic steatosis and markers of stellate cell activation with a trend to less liver fibrosis. This change in liver histology was associated with a reduction in insulin resistance. Unfortunately, many patients regained their weight at the end of the three months of weekly monitoring of calorie intake and exercise. Nevertheless, it is clear that simple lifestyle changes alone can potentially reduce both the morbidity (development of diabetes) and mortality (fibrosis leading to cirrhosis) related to hepatitis $\mathrm{C}$ infection.

These same authors previously showed that hepatic steatosis is common in individuals infected with hepatitis $\mathrm{C}$ and that the degree of steatosis and fibrosis correlates well with body mass index. ${ }^{2}$ It is also known that individuals infected with genotype 3 are more likely to have a fatty liver even when they are not overweight. ${ }^{3}$ A recent illustration demonstrated how eradication of hepatitis $\mathrm{C}$ virus following antiviral therapy can lead to disappearance of hepatic steatosis only to recur on viral relapse. ${ }^{4}$ Thus it would appear that hepatic steatosis in association with hepatitis $C$ is not simply secondary to obesity. In this current study, even those individuals with genotype 3 infection who were not overweight at the start had a diminution in hepatic steatosis on weight reduction, without the addition of antiviral therapy.

Although the patients in this study had normal fasting glucose levels, most were insulin resistant, as judged by the homeostasis model of assessment (HOMA) test. Fasting insulin levels correlated well with baseline BMI. Weight reduction led to an improvement in the HOMA score in the majority. Insulin resistance, also calculated using the HOMA method, has been noted by others to be common in individuals with hepatitis $\mathrm{C}$ and correlated with older age, obesity, severe liver fibrosis, and a family history of diabetes. ${ }^{5}$

In peripheral tissues, insulin normally downregulates the hormone sensitive lipase (HSL) enzyme responsible for hydrolysis of stored triglycerides from free fatty acids within adipocytes. In patients who are insulin resistant, this enzyme is no longer suppressed. In addition, counterregulatory hormones such as catecholamines, glucagon, and growth hormone are increased in response to increased circulating insulin levels. These counterregulatory hormones stimulate HSL to hydrolyse more triglycerides into free fatty acids, the end result being an increased flux of dietary and stored free fatty acids away from the adipose tissues and towards the liver. Unfortunately, Hickman et al did not measure free fatty acid levels before or after the weight reduction programme. Within the liver, insulin upregulates esterification of free fatty acids to triglycerides. Once the triglycerides are formed, insulin downregulates the secretory pathways, thus favouring increased storage of triglycerides in the cytosolic pool. Furthermore, free fatty acids can themselves upregulate the esterification pathway. The net result is a positive feedback cycle contributing to an ever increasing amount of free fatty acids and 
triglycerides in the liver. Thus portal hyperinsulinaemia leads to hepatic steatosis.

It is unfortunate that the authors used two methods to achieve weight reduction simultaneously. Exercise induced lowering of plasma insulin causes mobilisation of free fatty acids from the liver ${ }^{6}$ but the effect of exercise alone on hepatic steatosis has not been tried in individuals with non-alcoholic steatohepatitis or hepatitis C. Hickman et al were able to collect liver tissue both at baseline and again within three months of cessation of the 12 week weight reduction programme in 10 of the 19 individuals who participated in this study. Weight loss was associated with both a significant reduction in the grade of hepatic steatosis and a significant reduction in stellate cell activity, as judged by the extent of $\alpha$ smooth muscle actin staining. A significant reduction in liver fibrosis was not seen-not surprising over a six month period. These authors have provided some excellent photos of liver histology showing the marked changes in hepatic steatosis observed in 9/10 patients who underwent a second biopsy. However, fat is often focal and with this small number of paired biopsies, sampling error could still explain these changes although this is unlikely as $90 \%$ showed improvement of hepatic fat after weight loss. There is another study from Japan which also combined dietary restriction and an exercise programme in patients with fatty liver (uncomplicated by hepatitis C). They also demonstrated that a three month programme of weight reduction led to a significant reduction in hepatic steatosis but no change in fibrosis. ${ }^{7}$ These authors did not examine stellate cell activation. Clearly, the next experiment should be to establish whether exercise alone will induce similar changes.

There is still a lot that we do not know about hepatic steatosis and hepatitis C. These studies have suggested that the presence of fat in patients with hepatitis $\mathrm{C}$ is associated with markers of progressive liver disease in that fat was associated with increased stellate cell activation, but the mechanism by which this takes place is uncertain. It is possible that this occurs secondary to saturation of beta oxidation pathways within mitochondria which then leads to free fatty acids becoming more available to intracellular microsomes where they undergo lipid peroxidation. There are three main products of microsomal lipid peroxidation: malondialdehyde, 4-hydroxynonenal, and hydrogen peroxide. Malondialdehyde has been shown to activate stellate cells to produce fibrin, and may be responsible at least in part for liver fibrosis in patients with nonalcoholic steatohepatitis.

Recent data indicate that obesity is correlated with an impaired response to antiviral therapy in hepatitis C. ${ }^{8}$ It has been shown that the efficacy of "combination" therapy is in part related to the dose of ribavirin. This nucleoside analogue has a large volume of distribution and therefore body weight influences serum concentrations of ribavirin. Interferon, both standard and pegylated, has to be given by injection. Whereas standard interferon also has a large volume of distribution, as the size of the interferon molecule is increased (with pegylation), the volume of distribution diminishes so that body weight should not have such a marked effect on antiviral activity. However, it is possible that the site of the injection may influence absorption of IFN. In patients with diabetes, absorption of insulin differs according to the site of injection-for example, less in a slender arm and greater in an obese abdomen. Thus potentially weight loss could indirectly alter drug metabolism. This point has been discussed by Giannini and colleagues.' One wonders whether this is one explanation for impaired response to antiviral therapy in hepatitis C in African-Americans who have a high rate of central obesity and insulin resistance.

Hickman et al have opened yet another chapter in the complicated story of the mechanisms involved in disease progression and possibly to factors influencing resistance to therapy in patients with hepatitis $\mathrm{C}$. We have a lot more to learn!

Gut 2002;51:7-8

\section{Author's affiliation}

J Heathcote, Toronto Western Hospital, University Health Network, 399 Bathurst St, 6B Fell Pavilion, Room 172, Toronto, Ontario M5T 2S8, Canada; jenny.heathcote@utoronto.ca

\section{REFERENCES}

1 Hickman IJ, Clouston AD, Macdonald GA, et al. Effect of weight reduction on liver histology and biochemistry in patients with chronic hepatitis C. Gut 2002;51:89-94.

2 Hourigan LF, Macdonald GA, Purdi D, et al. Fibrosis in chronic hepatitis $C$ correlates significantly with body mass index and steatosis. Hepatology 1999;29:1215-19.

3 Rubia-Brandt L, Quadri R, Abid K, et al. Hepatocyte steatosis is a cytopathic effect of hepatitis $C$ virus genotype 3 . J Hepatol 2000;33:106-15.

4 Rubbia-Brandt L, Giostia E, Menthas G, et al. Expression of liver steatosis in hepatitis $C$ virus infection and pattern of response to alpha-interferon. J Hepatol 2001;35:307.

5 Petit JM, Bour JB, Galland-Jol C, et al. Risk factors for diabetes mellitus and early insulin resistance in chronic hepatitis C.J Hepatol 2001;35:279-83.

6 Romijin JA, Coyle EF, Sidossis LS, et al. Regulation of endogenous fat and carbohydrate metabolism in relation to exercise intensity and duration. Am J Physiol 1993;265: E380-91.

7 Ueno T, Sugawara H, Sujaku K, et al. Therapeutic effects of restricted diet and exercise in obese patients with fatty liver. J Hepatol 1997;27:103-7.

8 Zeuzem S, Feinman SV, Rasenack J, et al. Peg interferon alfa-2a in patients with chronic hepatitis C. N Engl J Med 2000;343: 166672 .

9 Giannini E, Ceppa P, Testa R. Steatosis in chronic hepatitis $\mathrm{C}$ : can weight reduction improve therapeutic efficacy? I Hepatol $2001 ; 35: 432-3$. 